\title{
Synthesis and Anti-inflammatory Activity of Some Novel Pyrazole Derivatives of Gallic Acid
}

\author{
S. ARUNKUMAR ${ }^{*}$, K. ILANGO, R. S. MANIKANDAN and N. RAMALAKSHMI ${ }^{\S}$ \\ Department of Pharmaceutical Chemistry, S.R.M. College of Pharmacy, \\ S.R.M University, Kattankulathur - 603203, Tamilnadu, India. \\ ${ }^{\S}$ Department of Pharmaceutical Chemistry, C.L. Baid Metha College of Pharmacy, \\ Thoraipakkam, Chennai - 600096, Tamilnadu, India. \\ arunkumarsubramani@yahoo.co.in
}

Received 12 March 2009; Accepted 22 April 2009

\begin{abstract}
In the present study, a new series of [5-substituted-3(phenylamino)-1 $\mathrm{H}$-pyrazol-1yl] (3,4,5-trihydroxyphenyl)-methanone (4a-j) have been synthesized. 3, 4, 5-Trihydroxy benzohydrazide (1) was synthesized from propyl gallate and hydrazine hydrate in presence of ethanol. Chalcones (2a-j) were synthesized from acetanilide and various aromatic aldehydes in presence of ethanol and sodium hydroxide solution. By refluxing the compound (1) and compounds (2a-j) in presence of ethanol yielded [5-substituted-3-(phenylamino)-4.5-dihydropyrazol-1yl] (3,4,5-trihydroxy phenyl)-methanone (3a-j). The final compounds [5-substituted-3-(phenylamino)-1 $H$-pyrazol-1yl] (3,4,5-trihydroxyphenyl)methanone (4a-j) were synthesized by treating compounds $(\mathbf{3 a - j})$ with bromine water. The synthesized compounds have been characterized by IR, ${ }^{1} \mathrm{HNMR}$ and Mass spectral data. The compounds were evaluated for in vivo anti-inflammatory activity by carrageenan induced paw edema test. In general all compounds were found to exhibit good anti-inflammatory activity.
\end{abstract}

Keywords: Gallic acid, Chalcones, Pyrazole, Characterization, Anti-inflammatory activity.

\section{Introduction}

Gallic acid is a strong natural antioxidant and have been reported to posses anti bacterial, antifungal $^{1}$, anti-inflammatory ${ }^{2}$ and anti cancer ${ }^{3,4}$. Propyl gallate is the propyl ester of gallic acid which is involved in synthesis as precursor and used as antioxidant ${ }^{5}$. Derivatives of pyrazoles are also possessing antibacterial ${ }^{6.7}$, antifungal ${ }^{8}$ and anti-inflammatory activity. Based upon the above promising biological activities, it was proposed to synthesize and 
characterize [5-substituted-3-(phenylamino)-1H-pyrazol-1yl] (3,4,5-trihydroxyphenyl)methanone (4a-j) by refluxing the 3,4,5-trihydroxybenzohydrazide (1) with various chalcones (2a-j) in the presence of ethanol. The in vivo anti-inflammatory activity, of synthesized compounds $(\mathbf{4} \mathbf{a}-\mathbf{j})$ was evaluated by carrageenan induced paw oedma in rats and the results are compared with standard drug diclofenac sodium.

\section{Experimental}

The melting points of the synthesized compounds were determined in open capillary tubes and were found uncorrected. The IR spectra of the synthesized compounds were recorded using $\mathrm{KBr}$ pellets in range of $4000-400 \mathrm{~cm}^{-1}$ on a Fourier Transform IR spectrometer. ${ }^{1}$ HNMR spectra were recorded on Bruker $400 \mathrm{MHz}$ ADVANCE ${ }^{1} \mathrm{HNMR}$ spectrometer. The chemical shifts were reported in parts per million downfield from tetramethylsilane (TMS). Mass spectra were recorded on LC-MS Shimadzu 2010A using dimethyl sulfoxide as solvent. Purity of the compounds was checked by thin layer chromatography using $n$-hexane and ethyl acetate in the ratio of 8:2. The physical constants of synthesized compounds are reported in Table 1.

Table 1. Physical constants of [5-substituted-3-(phenylamino)-1H-pyrazol-1yl] (3,4,5trihydroxy phenyl)-methanone (4a-j)

\begin{tabular}{llllll} 
& & & & \\
\hline Compd & Ar value & $\mathrm{M} . \mathrm{F}$ & & & \\
\hline $\mathbf{4 a}$ & $\mathrm{C}_{6} \mathrm{H}_{5}$ & $\mathrm{C}_{22} \mathrm{H}_{17} \mathrm{~N}_{3} \mathrm{O}_{4}$ & 60 & 110 & 0.86 \\
$\mathbf{4 b}$ & $4-\left(\mathrm{OCH}_{3}\right)-\mathrm{C}_{6} \mathrm{H}_{4}$ & $\mathrm{C}_{23} \mathrm{H}_{18} \mathrm{~N}_{3} \mathrm{O}_{5}$ & 64 & 115 & 0.88 \\
$\mathbf{4 c}$ & $2-\mathrm{NO}_{2}-\mathrm{C}_{6} \mathrm{H}_{4}$ & $\mathrm{C}_{22} \mathrm{H}_{16} \mathrm{~N}_{4} \mathrm{O}_{6}$ & 68 & 105 & 0.79 \\
$\mathbf{4 d}$ & $3-\mathrm{NO}_{2}-\mathrm{C}_{6} \mathrm{H}_{4}$ & $\mathrm{C}_{22} \mathrm{H}_{16} \mathrm{~N}_{4} \mathrm{O}_{6}$ & 70 & 115 & 0.75 \\
$\mathbf{4 e}$ & $2-\mathrm{Cl}-\mathrm{C}_{6} \mathrm{H}_{4}$ & $\mathrm{C}_{22} \mathrm{H}_{16} \mathrm{ClN}_{3} \mathrm{O}_{4}$ & 66 & 120 & 0.88 \\
$\mathbf{4 f}$ & $4-\mathrm{Cl}-\mathrm{C}_{6} \mathrm{H}_{4}$ & $\mathrm{C}_{22} \mathrm{H}_{16} \mathrm{ClN}_{3} \mathrm{O}_{4}$ & 68 & 105 & 0.86 \\
$\mathbf{4 g}$ & $2-\mathrm{OH}-\mathrm{C}_{6} \mathrm{H}_{4}$ & $\mathrm{C}_{22} \mathrm{H}_{17} \mathrm{~N}_{3} \mathrm{O}_{5}$ & 59 & 115 & 0.86 \\
$\mathbf{4 h}$ & $3-\mathrm{OH}-\mathrm{C}_{6} \mathrm{H}_{4}$ & $\mathrm{C}_{22} \mathrm{H}_{17} \mathrm{~N}_{3} \mathrm{O}_{5}$ & 55 & 120 & 0.88 \\
$\mathbf{4 i}$ & $4-\mathrm{N}\left(\mathrm{CH}_{3}\right)_{2} \mathrm{C}_{6} \mathrm{H}_{4}$ & $\mathrm{C}_{24} \mathrm{H}_{22} \mathrm{~N}_{4} \mathrm{O}_{4}$ & 58 & 115 & 0.84 \\
$\mathbf{4 j}$ & $3,4,6-\left(\mathrm{OCH}_{3}\right)_{3}-\mathrm{C}_{6} \mathrm{H}_{2}$ & $\mathrm{C}_{25} \mathrm{H}_{23} \mathrm{~N}_{3} \mathrm{O}_{7}$ & 63 & 105 & 0.83 \\
\hline
\end{tabular}

Preparation of chalcones (2a-j)

A mixture of acetanilide $(0.01$ mole $)$ and appropriate aldehyde $(0.01$ mole $)$ were added in solution of ethanol $(25 \mathrm{~mL})$ and $20 \%$ sodium hydroxide solution $(40 \mathrm{~mL})$. Solution was stirred for 3 to $4 \mathrm{~h}$ by using magnetic stirrer. The mixture was then kept in refrigerator overnight. Crystals of chalcones was washed with water until solution become neutral to litmus paper and recrystallised from ethanol to give $85 \%$ yield of the title compound (Scheme 1). Melting point $112^{\circ} \mathrm{C}$ 

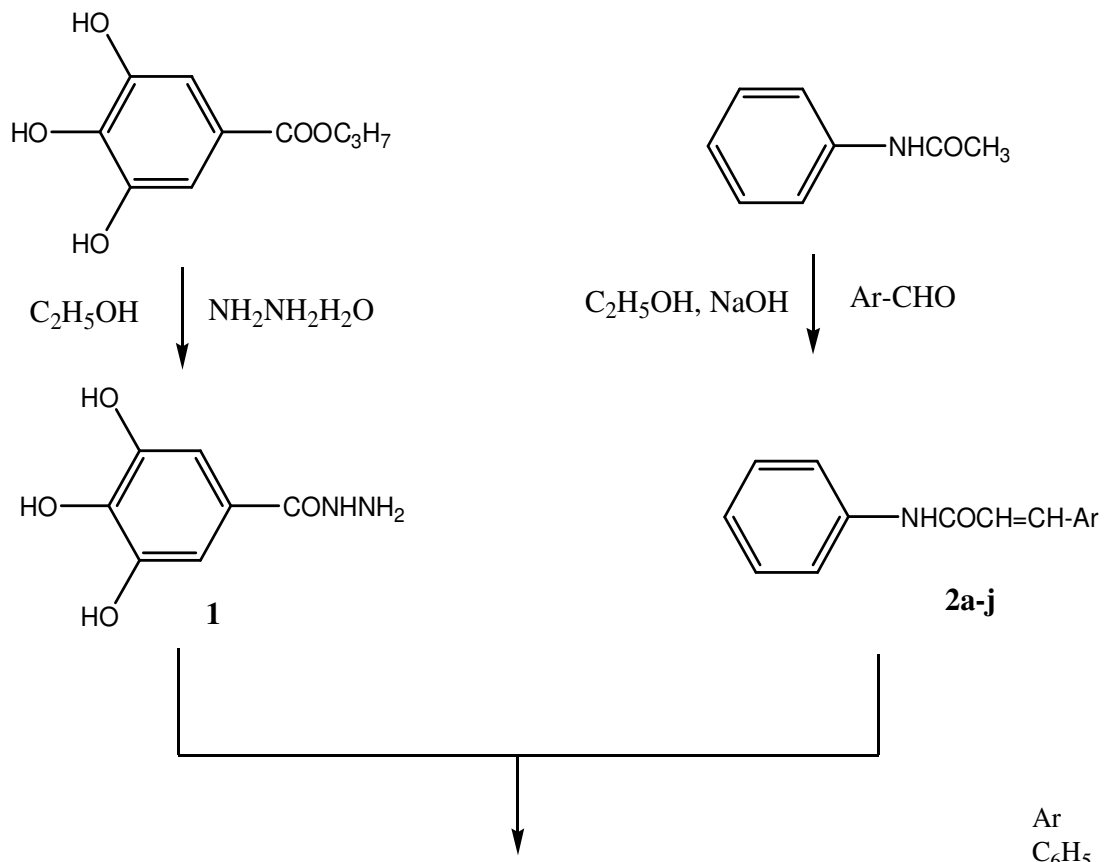

2a-j
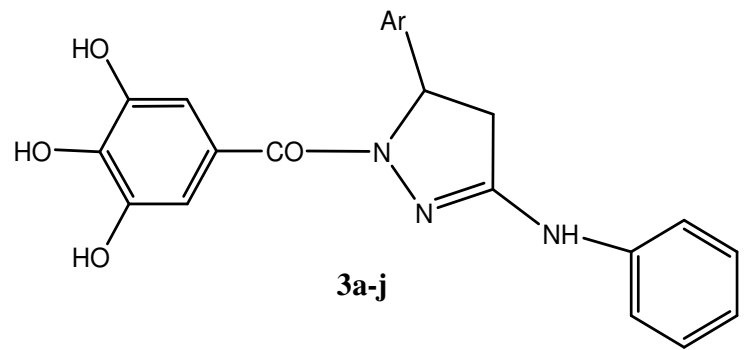

$\mathrm{Ar}$

$\mathrm{C}_{6} \mathrm{H}_{5}$

4- $\left(\mathrm{OCH}_{3}\right)-\mathrm{C}_{6} \mathrm{H}_{4}$

$2-\mathrm{NO}_{2}-\mathrm{C}_{6} \mathrm{H}_{4}$

$3-\mathrm{NO}_{2}-\mathrm{C}_{6} \mathrm{H}_{4}$

$2-\mathrm{Cl}-\mathrm{C}_{6} \mathrm{H}_{4}$

4- $\mathrm{Cl}-\mathrm{C}_{6} \mathrm{H}_{4}$

2-OH- $\mathrm{C}_{6} \mathrm{H}_{4}$

$3-\mathrm{OH}-\mathrm{C}_{6} \mathrm{H}_{4}$

$4-\mathrm{N}\left(\mathrm{CH}_{3}\right)_{2} \mathrm{C}_{6} \mathrm{H}_{4}$

3,4,6- $\left(\mathrm{OCH}_{3}\right)_{3}-\mathrm{C}_{6} \mathrm{H}_{2}$

$$
\mathrm{Br}_{2} / \mathrm{H}_{2} \mathrm{O}
$$

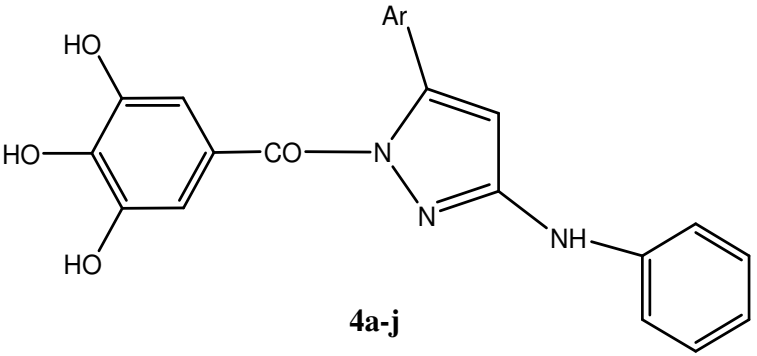

Scheme 1. Synthetic route to pyrazole derivatives of gallic acid. 
Preparation of (5-substituted-3-(phenylamino)-4,5-dihydropyrazol-1-yl)(3,4,5trihydroxy phenyl)methanone (3a-j)

A solution of the appropriate chalcones $(0.01$ mole $)$ in ethanol $(30 \mathrm{~mL})$ was refluxed with galloyal hydrazide $(0.01$ mole) (1) in presence of glacial acetic acid $(1 \mathrm{~mL})$ for $4 \mathrm{~h}$. The solution was cooled and diluted with water. The precipitated crude product was filtered and recrystallised from ethanol to give $80 \%$ yield of the title compound. Melting point is $120^{\circ} \mathrm{C}$.

Preparation of (5-substituted-3-(phenylamino)-1H-pyrazol-1yl)(3,4,5-trihydroxy phenyl)-methanone (4a-j)

A suspension of appropriate pyrazolines $(0.01$ mole $)(\mathbf{3 a - j})$ in water $(10 \mathrm{~mL})$ was treated with $10 \%$ bromine water with stirring until faint yellow color developed. The stirring was continued for $2 \mathrm{~h}$ and the crude pyrazole collected and recrystallised from methanol. The formations of compounds are confirmed by the difference in $\mathrm{m} . \mathrm{p}$ and $\mathrm{R}_{\mathrm{f}}$ values. The physical data are listed in Table 1.

Spectral study ${ }^{11}$ of (5-substituted-3-(phenylamino)-1H-pyrazol-1yl)(3,4,5-trihydroxy phenyl)-methanone (4a-j)

[5-Phenyl-3-(phenylamino)-1H-pyrazol-1yl] (3,4,5-trihydroxyphenyl) methanone (4a)

IR (KBr) $\left(\mathbf{c m}^{-1}\right): 3296.33(\mathrm{Ar}-\mathrm{OH}), 1663.46(\mathrm{C}=\mathrm{O}), 1312.67(\mathrm{C}-\mathrm{N})$.

${ }^{1}$ HNMR (DMSO-d (D) $^{1}$ (ppm): 3.417(s, 1H, NH), 5.11(s, 1H, OH), 7.45(m, Ar H).

Mass Spectra $(\mathrm{m} / \mathrm{z}): 387\left(\mathbf{M}^{+}\right), 215.90\left(\mathbf{B}^{+}\right)$.

[5-(4-Methoxyphenyl)-3-(phenylamino)-1H-pyrazol-1yl](3,4,5-trihydroxyphenyl)methanone (4b)

IR (KBr) $\left(\mathbf{c m}^{-1}\right)$ : $3200.60(\mathrm{Ar}-\mathrm{OH}), 1653.46(\mathrm{C}=\mathrm{O}), 1312.67(\mathrm{C}-\mathrm{N})$.

${ }^{1}$ HNMR (DMSO-d $) \delta(\mathrm{ppm}): 3.75(\mathrm{~s}, 1 \mathrm{H}, \mathrm{NH}), 5.01(\mathrm{~s}, 1 \mathrm{H}, \mathrm{OH}), 7.45(\mathrm{~m}, \mathrm{Ar} \mathrm{H})$.

Mass Spectra $(\mathrm{m} / \mathrm{z}): 419\left(\mathbf{M}^{+}\right), 215.75\left(\mathbf{B}^{+}\right)$.

[5-(2-Nitrophenyl)-3-(phenylamino)-1H-pyrazol-1yl](3,4,5-trihydroxyphenyl) methanone (4c)

IR (KBr) $\left(\mathbf{c m}^{-1}\right): 3286.33(\mathrm{Ar}-\mathrm{OH}), 1673.46(\mathrm{C}=\mathrm{O}), 1312.67(\mathrm{C}-\mathrm{N})$.

${ }^{1}$ HNMR (DMSO-d (D) $\delta$ (ppm):3.56(s, 1H, NH), 5.16(s, 1H, OH), 7.45(m, Ar H).

Mass Spectra $(\mathrm{m} / \mathrm{z}): 435\left(\mathrm{M}^{+}\right), 213.90\left(\mathrm{~B}^{+}\right)$.

[5-(3-nitrophenyl)-3-(phenylamino)-1H-pyrazol-1yl](3,4,5-trihydroxyphenyl) methanone (4d)

IR (KBr) $\left(\mathbf{c m}^{-1}\right): 3458.60(\mathrm{Ar}-\mathrm{OH}), 1654.18(\mathrm{C}=\mathrm{O}), 1322.36(\mathrm{C}-\mathrm{N})$.

${ }^{1}$ HNMR (DMSO-d (D) $\delta(\mathrm{ppm}): 3.44(\mathrm{~s}, 1 \mathrm{H}, \mathrm{NH}), 5.12(\mathrm{~s}, 1 \mathrm{H}, \mathrm{OH}), 7.45(\mathrm{~m}, \mathrm{Ar} \mathrm{H})$.

Mass Spectra $(\mathrm{m} / \mathrm{z}): 432\left(\mathbf{M}^{+}\right), 215.90\left(\mathbf{B}^{+}\right)$.

[5-(2-chlorophenyl)-3-(phenylamino)-1H-pyrazol-1yl] (3,4,5-trihydroxyphenyl)methanone (4e)

IR (KBr) (cm $\left.\mathbf{c m}^{-1}\right)$ : $3283.68(\mathrm{Ar}-\mathrm{OH}), 1659.00(\mathrm{C}=\mathrm{O}), 1320.32(\mathrm{C}-\mathrm{N})$.

${ }^{1}$ HNMR (DMSO-d $\left.{ }_{6}\right) \delta(\mathrm{ppm}): 3.43(\mathrm{~s}, 1 \mathrm{H}, \mathrm{NH}), 5.10(\mathrm{~s}, 1 \mathrm{H}, \mathrm{OH}), 7.45(\mathrm{~m}, \mathrm{Ar} \mathrm{H})$.

Mass Spectra $(\mathrm{m} / \mathrm{z}): 423\left(\mathrm{M}^{+}\right), 215.80\left(\mathbf{B}^{+}\right)$.

[5-(4-chlorophenyl)-3-(phenylamino)-1H-pyrazol-1yl](3,4,5-trihydroxyphenyl)methanone (4f)

IR (KBr) $\left(\mathbf{c m}^{-1}\right): 3296.33(\mathrm{Ar}-\mathrm{OH}), 1673.46(\mathrm{C}=\mathrm{O}), 1312.66(\mathrm{C}-\mathrm{N})$.

${ }^{1}$ HNMR (DMSO-d ${ }_{6}$ ) $\delta$ (ppm): 3.30(s, 1H, NH), 4.92(s, 1H, OH), 7.45(m, Ar H).

Mass Spectra $(\mathrm{m} / \mathrm{z}): 423\left(\mathrm{M}^{+}\right), 213.70\left(\mathrm{~B}^{+}\right)$. 
[5-(2-hydroxyphenyl)-3-(phenylamino)-1H-pyrazol-1yl](3,4,5-trihydroxyphenyl) methanone $(4 \mathrm{~g})$

IR (KBr) $\left(\mathbf{c m}^{-\mathbf{1}}\right)$ : $3254.06(\mathrm{Ar}-\mathrm{OH}), 1665.81(\mathrm{C}=\mathrm{O}), 1313.86(\mathrm{C}-\mathrm{N})$.

${ }^{1}$ HNMR (DMSO-d $\left.{ }_{6}\right) \delta$ (ppm): 3.21(s, 1H, NH), 4.97(s, 1H, OH), 7.45(m, Ar H).

Mass Spectra $(\mathrm{m} / \mathrm{z}): 401\left(\mathbf{M}^{+}\right), 215.90\left(\mathbf{B}^{+}\right)$

[5-(3-hydroxyphenyl)-3-(phenylamino)-1H-pyrazol-1yl] (3,4,5-trihydroxyphenyl) methanone (4h)

IR (KBr) $\left(\mathbf{c m}^{-1}\right)$ : $3200.00(\mathrm{Ar}-\mathrm{OH}), 1659.46(\mathrm{C}=\mathrm{O}), 1312.67(\mathrm{CN})$.

${ }^{1}$ HNMR (DMSO-d $\left.{ }_{6}\right) \delta(\mathrm{ppm}): 3.12(\mathrm{~s}, 1 \mathrm{H}, \mathrm{NH}), 5.21(\mathrm{~s}, 1 \mathrm{H}, \mathrm{OH}), 7.45(\mathrm{~m}, \mathrm{Ar} \mathrm{H})$.

Mass Spectra $(\mathrm{m} / \mathrm{z}): 405\left(\mathbf{M}^{+}\right), 213.95\left(\mathbf{B}^{+}\right)$.

[5-(4-(dimethylamino)phenyl)-3-(phenylamino)-1H-pyrazol-1yl](3,4,5-trihydroxyphenyl)methanone (4i)

IR (KBr) $\left(\mathbf{c m}^{-1}\right)$ : $3296.33(\mathrm{Ar}-\mathrm{OH}), 1663.46(\mathrm{C}=\mathrm{O}), 1312.67(\mathrm{CN})$.

${ }^{1}$ HNMR (DMSO-d $\left.{ }_{6}\right) \delta(\mathrm{ppm}): 2.41\left(\mathrm{~s}, 6 \mathrm{H}, \mathrm{N}\left(\mathrm{CH}_{3}\right), 3.32(\mathrm{~s}, 1 \mathrm{H}, \mathrm{NH}), 5.00(\mathrm{~s}, 1 \mathrm{H}, \mathrm{OH})\right.$, 7.45(m, Ar H).

Mass Spectra $(\mathrm{m} / \mathrm{z}): 435\left(\mathbf{M}^{+}\right), 215.85\left(\mathbf{B}^{+}\right)$.

[3-(phenylamino)-5-(3,4,5-trimethoxyphenyl)-1H-pyrazol-1yl] (3,4,5-trihydroxyphenyl) methanone $(4 \mathbf{j})$

IR (KBr) $\left(\mathbf{c m}^{-1}\right)$ : $3296.33(\mathrm{Ar}-\mathrm{OH}), 1663.46(\mathrm{C}=\mathrm{O}), 1312.67(\mathrm{CN})$.

${ }^{1}$ HNMR (DMSO-d $) \delta$ (ppm): 3.43(s, 1H, NH), 5.10(s, 1H, OH), 7.45(m, Ar H).

Mass Spectra $(\mathrm{m} / \mathrm{z}): 475\left(\mathrm{M}^{+}\right), 215.70\left(\mathrm{~B}^{+}\right)$.

\section{Results and Discussion}

The structures of newly synthesized compounds have been authentically established by their TLC, m.p, IR, ${ }^{1}$ HNMR and Mass spectral analysis. The compounds (4a-j) were obtained by reacting chalcones and galloyl hydrazide in the presence of ethanol. The yield was found to be satisfactory in the range of 50-70\%. All the compounds exhibited significant antiinflammatory activity. Among the ten compounds $\mathbf{4 b}, \mathbf{4 e}$, and $\mathbf{4 j}$ showed comparable activity while compounds $\mathbf{4 a}, \mathbf{4 c}, \mathbf{4 d}, \mathbf{4 g}, \mathbf{4 h}$, and $\mathbf{4 i}$ showed less activity when compared with standard drug diclofenac sodium.

\section{Anti-inflammatory activity}

All the synthesized compounds were screened for in vivo anti-inflammatory activity by carrageenan induced paw edema test in rats ${ }^{9,10}$. Diclofenac sodium $(20 \mathrm{mg} / \mathrm{kg})$ was administered as standard drug for comparison. Rats were divided in to 12 groups each group containing 4 rats. Group I was treated with tween-80 (1\%) suspension which served by vehicle control. Group II to XI were treated with the suspension of the test compounds (4a-j) at a dose of $20 \mathrm{mg} / \mathrm{kg}$. Group XII was administered with standard drug Diclofenac sodium. After 30 minutes, the animals were injected with $0.1 \mathrm{~mL}$ of carrageenan $(1 \% \mathrm{w} / \mathrm{v})$, in the sub planter region of left hind paw of rats. The paw volume was measured using the mercury displacement technique with the help of a plethysmometer after $2 \mathrm{~h}$ and $4 \mathrm{~h}$ of carrageenan injection. The formula used for calculating the percentage inhibition of edema is

$$
\% \text { Inhibition }=[1-(\mathrm{Vt} / \mathrm{Vc})] * 100
$$

Where, $\mathrm{Vt}$ and $\mathrm{Vc}$ are the mean relative changes in the volume of paw edema in the test and control respectively. The results are given in Table 2 . 
Table 2. Anti-inflammatory activity of [5-substituted-3-(phenylamino)-1H-pyrazol-1yl] (3,4,5-trihydroxy phenyl) methanone (4a-j).

\begin{tabular}{cllcc}
\hline \multirow{2}{*}{ Group } & Edema & volume & \multicolumn{2}{c}{$\%$. inhibition } \\
& $2 \mathrm{~h}$ & $4 \mathrm{~h}$ & $2 \mathrm{~h}$ & $4 \mathrm{~h}$ \\
\hline $1 \%$ Tween-80 & $0.47 \pm 0$. & $0.50 \pm 0.02$ & - & - \\
$\mathbf{7 a}$ & $0.26 \pm 0$. & $0.23 \pm 0.01$ & $43.33 \pm 2.15$ & $38.32 \pm 2.86$ \\
$\mathbf{7 b}$ & $0.21 \pm 0$. & $0.25 \pm 0.03$ & $54.86 \pm 1.42$ & $49.69 \pm 3.80$ \\
$\mathbf{7 c}$ & $0.23 \pm 0$. & $0.27 \pm 0.02$ & $54.24 \pm 2.77$ & $44.74 \pm 2.73$ \\
$\mathbf{7 d}$ & $0.27 \pm 0$. & $0.31 \pm 0.02$ & $41.26 \pm 2.09$ & $36.49 \pm 2.68$ \\
$\mathbf{7 e}$ & $0.22 \pm 0$. & $0.26 \pm 0.02$ & $52.73 \pm 3.42$ & $47.91 \pm 1.40$ \\
$\mathbf{7 f}$ & $0.22 \pm 0$. & $0.27 \pm 0.03$ & $51.92 \pm 1.88$ & $45.27 \pm 4.27$ \\
$\mathbf{7 g}$ & $0.26 \pm 0$. & $0.30 \pm 0.02$ & $43.86 \pm 1.67$ & $40.22 \pm 1.97$ \\
$\mathbf{7 h}$ & $0.25 \pm 0$. & $0.29 \pm 0.02$ & $46.99 \pm 2.26$ & $41.22 \pm 3.01$ \\
$\mathbf{7 i}$ & $0.23 \pm 0$. & $0.27 \pm 0.02$ & $50.31 \pm 1.81$ & $45.82 \pm 2.68$ \\
$\mathbf{7 j}$ & $0.24 \pm 0$. & $0.27 \pm 0.02$ & $49.14 \pm 2.83$ & $45.27 \pm 1.04$ \\
Diclofenac sodium & $0.21 \pm 0$. & $0.23 \pm 0.01$ & $55.5 \pm 1.97$ & $53.20 \pm 0.38$ \\
\hline
\end{tabular}

All values are mean \pm SEM values using 4 animals in each group.

\section{Conclusions}

The yield of all 5-substituted-3-(phenylamino)-1H-pyrazol-1yl)(3,4,5-trihydroxy phenyl)methanone (4a-j) were found to be range of 50-70 \% and purity was confirmed by melting point and TLC. Characteristic IR bands show several functional vibrations, which confirm the completion of reaction. The structures of the compounds synthesized were confirmed by ${ }^{1}$ HNMR and Mass spectral studies. The compounds were also screened for their antiinflammatory activity by carrageenan induced paw edema method using Diclofenac sodium as standard. The observation revealed that compounds $(\mathbf{4 b}),(\mathbf{4 e})$ and $(\mathbf{4 j})$ showed good antiinflammatory activity with percentage inhibition of 55.60, 55.89 and 55.60 respectively.

\section{References}

1. Arunkumar S, Ramalakshmi N, Saraswathy T and Aruoly L, Indian J Heterocycl Chem., 2006, 16, 29-32.

2. Sang-Hyun Kim, Chang-Duk Jun, Kyongho Suk, Byung-Ju Choi, Hyunjeung Lim, Seunja Park, Seung Ho Lee, Hye-Young Shin, Dae-Keun Kim and Tae-Yong Shin, Toxicol Sci., 2006, 91, 123-131.

3. Indap M A, Radhika S, Motiwale L and Rao K, Indian J Pharm Sci., 2006, 68, 470-474.

4. Inoue M, Suzuki R, Sakaguchi N, Li Z, Takeda T, Ogihara Y, Jiang BY, and Chen Y, Biol Pharm Bull., 1995, 18, 1526-1530.

5. Jain R, Pandya P, Bhadauria J and Tomar S, J Indian Chem Soc., 2000, 77, 42-43.

6. Jain V K and Rao J T, Indian Drugs, 2004, 7, 334-338.

7. The Merck Index, $13^{\text {th }}$ Ed., Merck \& Co., Inc., Whitehouse station: NJ USA, 2001, 772.

8. Gupta U, Sareen V, Khatri V and Chug S, Indian J Heterocycl Chem., 2004, 14, 351.

9. Mohd Amir, Shah Alam Khan and Khan M, Indian J Heterocycl Chem., 2001, 11, 55-58.

10. Mohamed S M, Khalile M and Ismile A A, J Indian Chem Soc., 2005, 82, 833-837.

11. Silverstein R, Spectroscopic identification of organic compounds; $6^{\text {th }}$ Edn., John Wiley \& Sons, Inc.: Newyork, 1991, 60-70, 119-176, 191-200. 


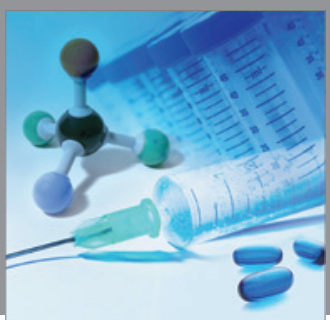

International Journal of

Medicinal Chemistry

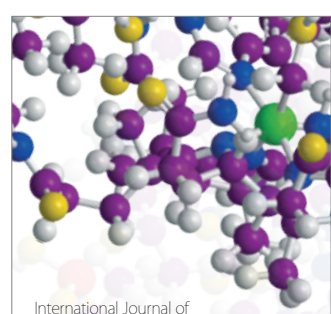

Carbohydrate Chemistry

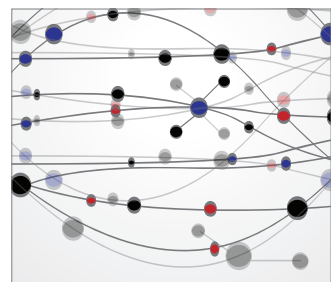

The Scientific World Journal
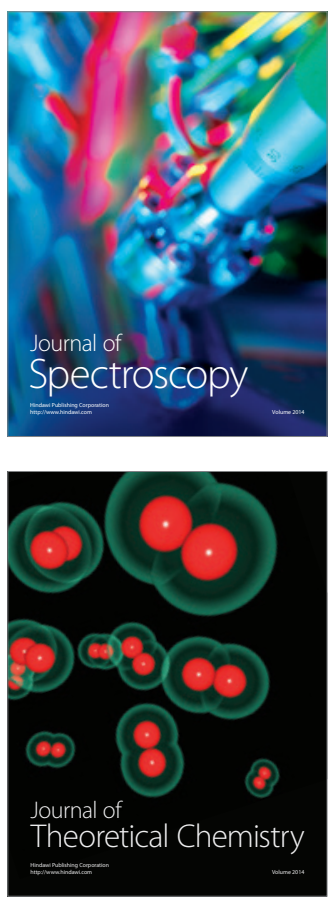
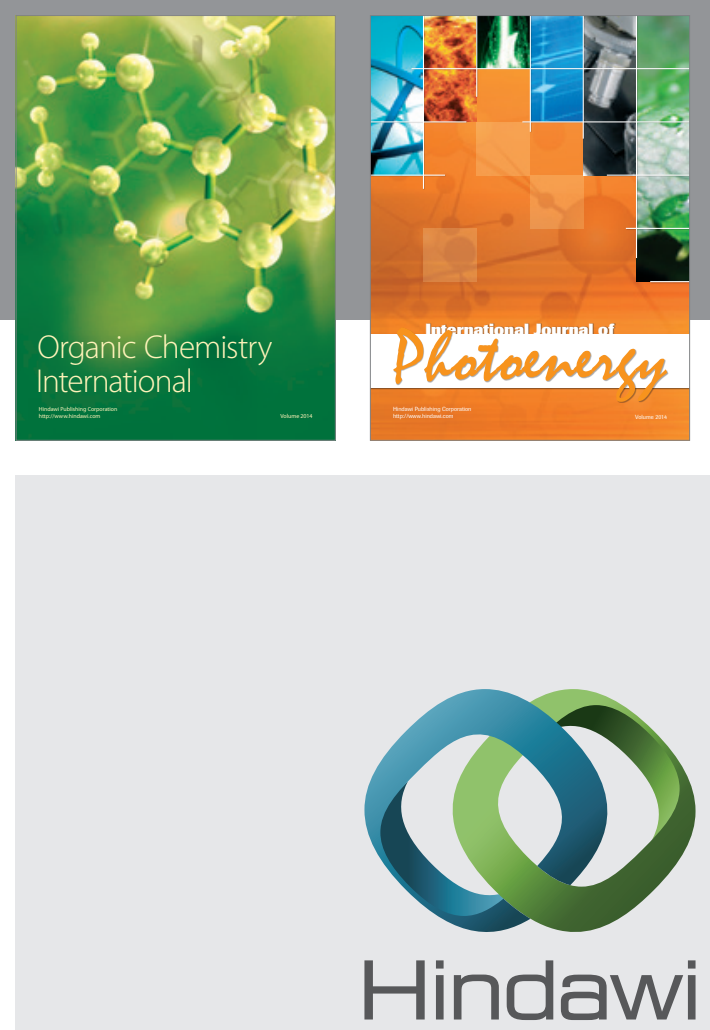

Submit your manuscripts at

http://www.hindawi.com
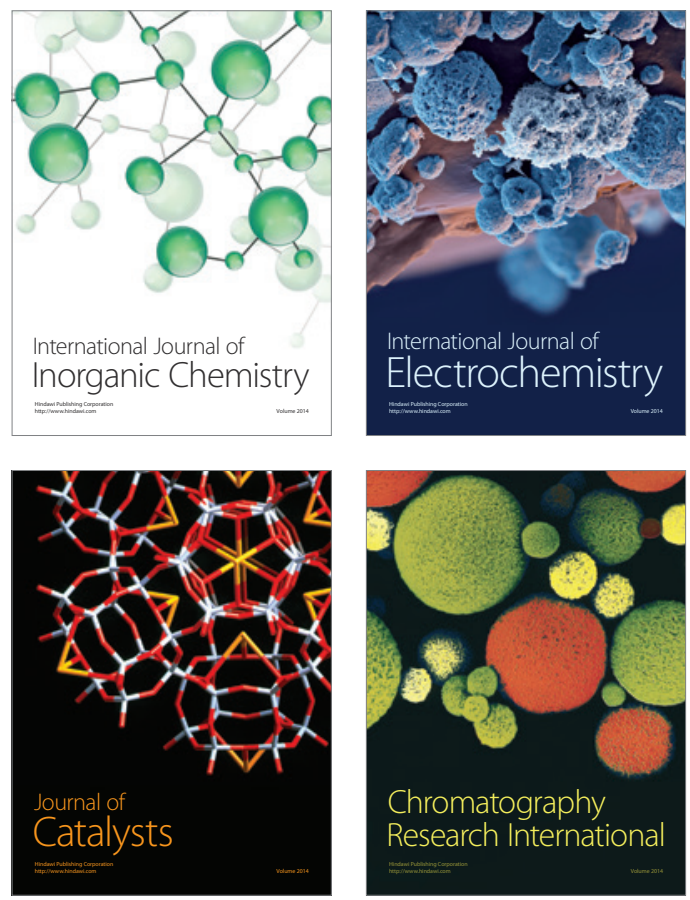
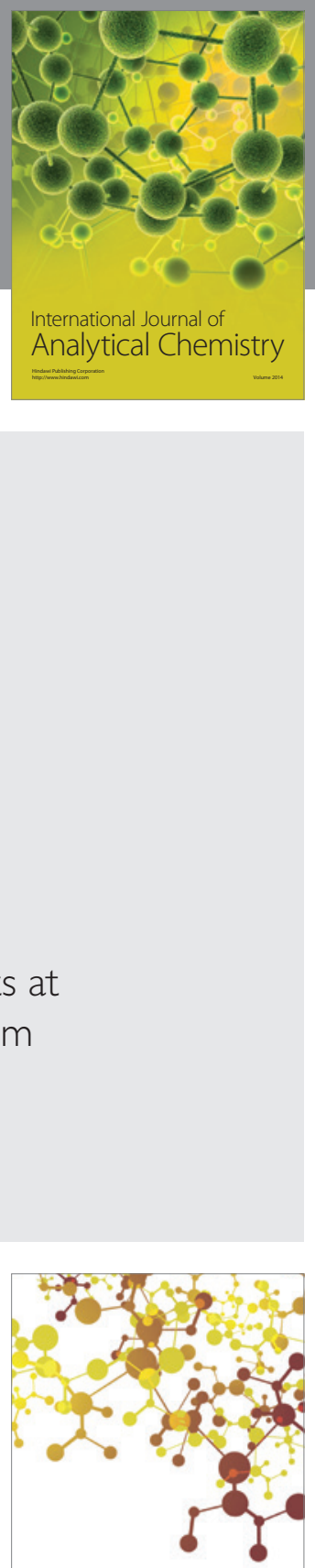

Journal of

Applied Chemistry
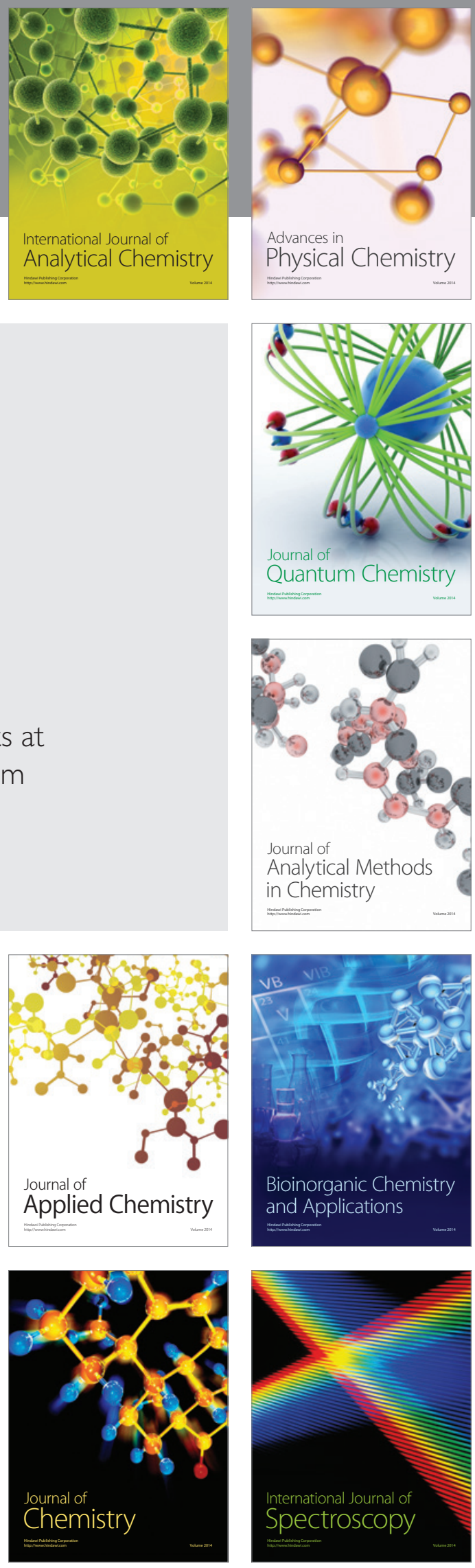